\title{
Pneumatic Muscles Actuated Lower-Limb Orthosis Model Verification with Actual Human Muscle Activation Patterns
}

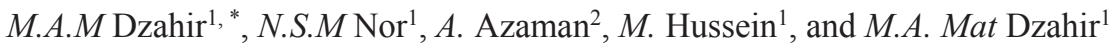 \\ ${ }^{1}$ Department of Applied Mechanics \& Design, Faculty of Mechanical Engineering, UTM, Malaysia \\ ${ }^{2}$ Department of Biotechnology \& Medical Engineering, Faculty of Biomedical Engineering and \\ Health Science, UTM, Malaysia
}

\begin{abstract}
A review study was conducted on existing lower-limb orthosis systems for rehabilitation which implemented pneumatic muscle type of actuators with the aim to clarify the current and on-going research in this field. The implementation of pneumatic artificial muscle will play an important role for the development of the advanced robotic system. In this research a derivation model for the antagonistic mono- and bi-articular muscles using pneumatic artificial muscles of a lower limb orthosis will be verified with actual human's muscle activities models. A healthy and young male 29 years old subject with height $174 \mathrm{~cm}$ and weight $68 \mathrm{~kg}$ was used as a test subject. Two mono-articular muscles Vastus Medialis (VM) and Vastus Lateralis (VL) were selected to verify the mono-articular muscle models and muscle synergy between anterior muscles. Two biarticular muscles Rectus Femoris (RF) and Bicep Femoris (BF) were selected to verify the bi-articular muscle models and muscle co-contraction between anterior-posterior muscles. The test was carried out on a treadmill with a speed of $4.0 \mathrm{~km} / \mathrm{h}$, which approximately around $1.25 \mathrm{~m} / \mathrm{s}$ for completing one cycle of walking motion. The data was collected for about one minute on a treadmill and 20 complete cycles of walking motion were successfully recorded. For the evaluations, the mathematical model obtained from the derivation and the actual human muscle activation patterns obtained using the surface electromyography (sEMG) system were compared and analysed. The results shown that, high correlation values ranging from 0.83 up to 0.93 were obtained in between the derivation model and the actual human muscle's model for both mono- and biarticular muscles. As a conclusion, based on the verification with the sEMG muscle activities data and its correlation values, the proposed derivation models of the antagonistic mono- and bi-articular muscles were suitable to simulate and controls the pneumatic muscles actuated lower limb orthosis.
\end{abstract}

\footnotetext{
*Corresponding author: azuwan@mail.fkm.utm.my / azuwan@,fkm.utm.my
} 


\section{Introduction}

It is a general assumption that pneumatic muscles will play an important role in the development of assistive rehabilitation robotics system. In the last decade, the development of the pneumatic muscle actuated lower limb orthosis system was relatively slow compared to the motorized orthosis system. However, in recent years, the interest in this field had grown exponentially mainly due to the demand on a much compliant human-robotics system and advantageous attributes of the pneumatic muscles. Based on the review study, it could be understood that the suitable control schemes and strategies have yet to be found. Therefore, in this study, a novel control scheme and strategy for manipulating the anteriorposterior mono- and bi-articular muscle actuators was proposed using simplified pneumatic muscles actuated lower limb orthosis derivation model and sEMG muscle activities of the human lower limb muscles. From the literature review analysis on the pneumatic muscle actuated lower limb orthosis, it shows that around 16 prototyped had been evaluated based on the development of different orthosis systems in last 10 years [1-11].

Moreover, most of the existing pneumatic muscle actuated lower limb systems antagonistically controlled the leg orthosis with anterior-posterior mono-articular actuators at the hip, knee, or ankle joints [12 - 25]. However, compared to most of the published research articles, only few articles had introduced anterior-posterior bi-articular muscle actuators to simultaneously control the lower limb orthosis along with anterior-posterior mono-articular muscle actuators [29, 30]. The design of the proposed lower limb orthosis model was based on the human musculoskeletal system. This human musculoskeletal system consisted of different mono- and bi-articular muscles to support the movement of the joints. The muscles could be group into two different types of muscles which are monoarticular muscles (i.e., Gluteus Maximus, Gluteus Minimus, Gluteus Medius, Vastus Medialis and Vastus Lateralis) and bi-articular muscles (i.e., Gastrocnemius, Rectus Femoris, and Bicep Femoris). These antagonistic muscles (i.e., agonist and antagonist) exhibit co-contraction movements in order to initiate and precisely move the human exoskeleton system. The co-contraction of the human muscles also contributes in increasing the stiffness at the joints and improves the human balance. In addition, these set of movements also to prevent an injury at the joints. This concept will be utilised to design the control scheme and strategy of the lower limb leg orthosis.

Meanwhile, different suggestions and remarks also have been reached by the researchers to improve the design and control system of the pneumatic muscle actuated lower-limb orthosis, which why it might be concluded that the researchers' interest has been shifted to the implementation of the natural type of compliant actuators [2 - 11]. Although lots of researches have been investigated regarding the co-contraction movements of human antagonistic muscles. However, their model implementation in controlling the antagonistic muscle actuators of lower-limb orthosis has not been completely discovered [12 - 25]. In addition, research study which focuses on the implementation of mono- and bi-articular actuators using pneumatic muscles for the lower-limb rehabilitation orthosis has yet to be extensively investigated [1,29]; thus, simply actuating the actuators might not give a good result on the joint's stiffness and stability of the lower-limb leg orthosis and its joint trajectories. Therefore, based on the related research findings, the simultaneous cocontractively like movements between the anterior and posterior actuators could be considered within the control system strategy [26 - 30].

Even though a considerable amount of work has now been done, this field is still rapidly evolving. The issue of which are the most effective control algorithms is still wide open. However, the randomized controlled trials are still necessary for identifying the suitable control algorithms even though it is expensive and time-consuming. Therefore, the purpose of this study is to validate the derivation model of the pneumatic muscle actuated lower 
limb orthosis with the actual human's muscle activities model during walking motion. Where, it is to identify the new method for controlling the orthosis system using the cocontractively control of the antagonistic mono- and bi- articular pneumatic muscle actuators.

\section{Model derivation of the mono- and bi-articular muscle actuators}

The contraction patterns of the antagonistic mono- and bi-articular artificial muscles of the pneumatic muscle actuated lower limb orthosis were found to be opposite to each other in between its anterior and posterior muscles [1]. Therefore, co-contraction models that generate input patterns for the antagonistic mono- and bi-articular actuators could be theoretically derived. To complete this theory, the model of the leg orthosis should be simplified before all the information could be group together, and then correlate all the important parameters into a mathematical equation as shown in Figure 1. The obtained model equations correlate the contraction of each of the antagonistic actuators with the positional data of hip and knee joints. The model derivation of the anterior-posterior contraction patterns could be referred from the previous journal publication [29]. Based on the derived mathematical model, the contraction of antagonistic mono-articular actuators for hip joint has a linear relationship with the hip joint angle. The contraction was also similar when compared at the antagonistic mono-articular actuators for the knee joint. Interestingly, the antagonistic bi-articular actuators have a linear relationship with a total of hip and knee joint angle as it manages the two joints simultaneously. The general idea for this model derivation was obtained based on the information that could be interpreted from the reference input data of the hip and knee joints. 


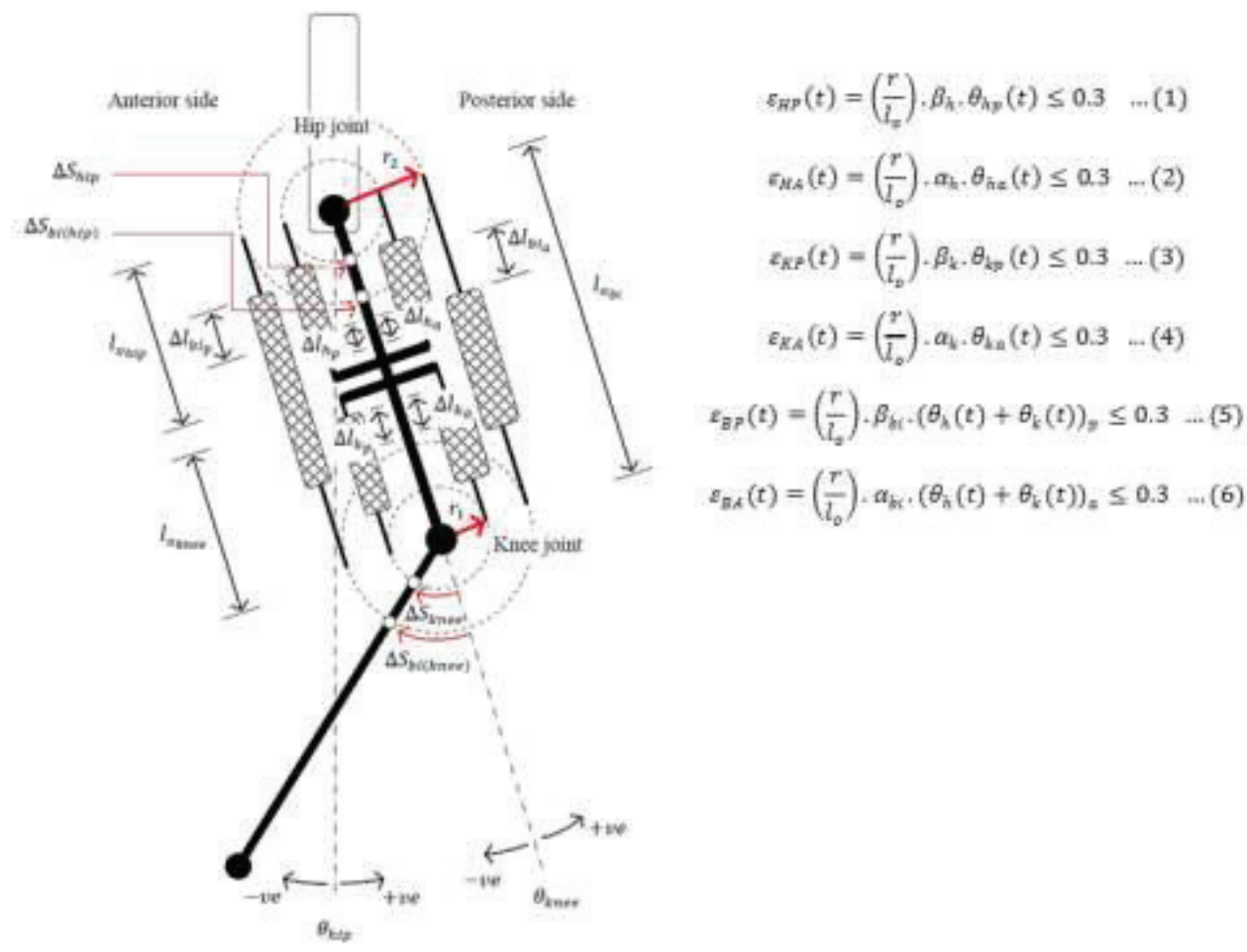

Fig. 1. Pneumatic muscle actuated lower limb orthosis simplified model for mono- and bi-articular muscles between hip and knee joints; where, $\varepsilon_{H A}$ and $\varepsilon_{H P}$ are the anterior-posterior muscle contraction of the hip joint; $\varepsilon_{K A}$ and $\varepsilon_{K P}$ are the anterior-posterior muscle contraction of the knee joint; $\varepsilon_{B A}$ and $\varepsilon_{B P}$ are the anterior-posterior muscle contraction of the bi-articular muscle (hip and knee joint); $\alpha$ and $\beta$ are muscle activation level for the anterior-posterior muscles; $\theta_{H}$ and $\theta_{K}$ are hip and knee joints.

\section{Methodology}

The first part of this research is to obtain a derivation model equations of the pneumatic muscle actuated lower limb orthosis as shown in Figure 1. These model equations represent the contraction value of each of the antagonistic mono- and bi-articular pneumatic muscles of the orthosis system. By introducing an input pattern of hip and knee joints during one complete cycle of walking motion into the mathematical model equations, the contractionexpansion value of each of anterior-posterior mono- and bi-articular pneumatic muscles can be obtained, and the verified with the actual human's muscle activities model. The second part of the research is to obtain sEMG muscle activities data model for both mono- and biarticular muscles of human's lower limb using fifth order of polynomial equation. A healthy and young male 29 years old subject with height $174 \mathrm{~cm}$ and weight $68 \mathrm{~kg}$ was used as a test subject. The subject is an active sport player and has a strong lower limb muscles. Two mono-articular muscles which are Vastus Medialis (VM) and Vastus Lateralis (VL) were used to verify the mono-articular muscle models and muscle synergy between anterior muscles. Two bi-articular muscles Rectus Femoris (RF) and Bicep Femoris (BF) were used to verify the bi-articular muscle models and muscle co-contraction between anteriorposterior muscles. The sEMG system (TMSi system, Netherland) was used to obtain sEMG muscle activation data of four muscles during walking motion. The test was carried out on a 
treadmill with a speed of $4.0 \mathrm{~km} / \mathrm{h}$, which approximately around $1.25 \mathrm{~m} / \mathrm{s}$ for completing one cycle of walking motion. Four sEMG electrodes were placed at the VM, VL, RF and $\mathrm{BF}$ muscles. The data was collected for about one minute on a treadmill and 20 complete cycles of walking motion were successfully recorded. The data were processed, filtered, and rectified to remove all the unrequired noise. A fifth order polynomial equations were used correlate the data into a model equations. The final part of the research is to verify and correlate the obtained derivation model with the actual human lower limb muscle's model for both mono- and bi-articular muscles.

\section{EMG muscle activities}

It is strongly believed that the human antagonistic muscles activate simultaneously, when one muscle (i.e., agonist or antagonist) is in contraction the other muscle (i.e., antagonist or agonist) will be in expansion and vice versa. We define this as muscle co-contraction or muscle synergy. Muscle co-contraction refers to when any movement occurs which involved two sets of muscles working around joint. Normally, the muscles on one side of the joint must relax so that the muscles on the other side can contract. However, in cocontraction is defined as both sets of muscles contract. Moreover, the definition of the cocontraction also could be explained as both muscles were simultaneously made contractionexpansion movements. When the muscles on one side of the joint are in contraction, the opposite muscles will not be in completely relaxed state. However, they are still in contraction but less than the opposite muscles. The muscle synergy refers to the interaction of two or more sets of muscles to produce a combined effect greater than the sum of their separate effects. Therefore, the result of this research study would verify the muscle activities of the human lower limb muscles both on the antagonistic mono- and bi-articular muscles. However, not all lower limb muscle could be easily obtained using sEMG muscle activity sensors due to small muscle's site and weak muscle activation. Thus only VM, VL, $\mathrm{RF}$, and BF muscles were selected to represent the anterior-posterior mono- and bi-articular muscles.

\section{Results and discussion}

For the result evaluations, VM and VL muscles were used to verify the anterior monoarticular muscle model while, RF and BF muscles were used to verify anterior-posterior biarticular muscle models. In this research, it was crucial to obtain good correlation value for the sEMG muscle activities of the antagonistic bi-articular muscles because these muscles activities were more complex when compared to the mono-articular muscles. Figure 2 shows the evaluation of the anterior mono-articular muscle model obtained from the mathematical derivation with VM (alone) and VM-VL (combined) muscle activities models. While, Figure 3 shows the evaluation of the anterior-posterior bi-articular muscle models obtained from the mathematical derivation with RF and BF muscle activities models.

Based on the results, it shows that the derivation models for both anterior-posterior mono- and bi-articular muscles were comparable to the actual muscles activities for the $\mathrm{VM}, \mathrm{VL}, \mathrm{RF}$, and BF lower-limb muscles. In addition, the patterns of the sEMG muscle activities data were highly correspond with the derived antagonistic mono- and bi-articular artificial muscle models. These results might indicate that the muscle activities of the antagonistic mono- and bi-articular muscles followed a certain patterns which can be defined by suitable model derivation. The evaluation on the VM and VM-VL muscle activity patterns which were both anterior mono-articular muscle proved that muscle 
synergy was occurred between same anterior or posterior muscles. Figure 2 shows that there were an improvement in between the muscle activities data patterns when we compared the VM muscle activities alone and a combination of VM-VL muscle activities with the derivation models. Furthermore, the evaluation on the RF and BF muscle activities patterns proved that the human lower limb muscles exhibit co-contraction movements between anterior and posterior muscles. However, an existing delay less than 0.40 seconds occur in-between the activation of the anterior and posterior sEMG muscle activities. Albeit that, the patterns of the sEMG muscle activities for VM, VL, RF, and BF muscles were still agreeing with the derived antagonistic mono- and bi-articular muscle models.
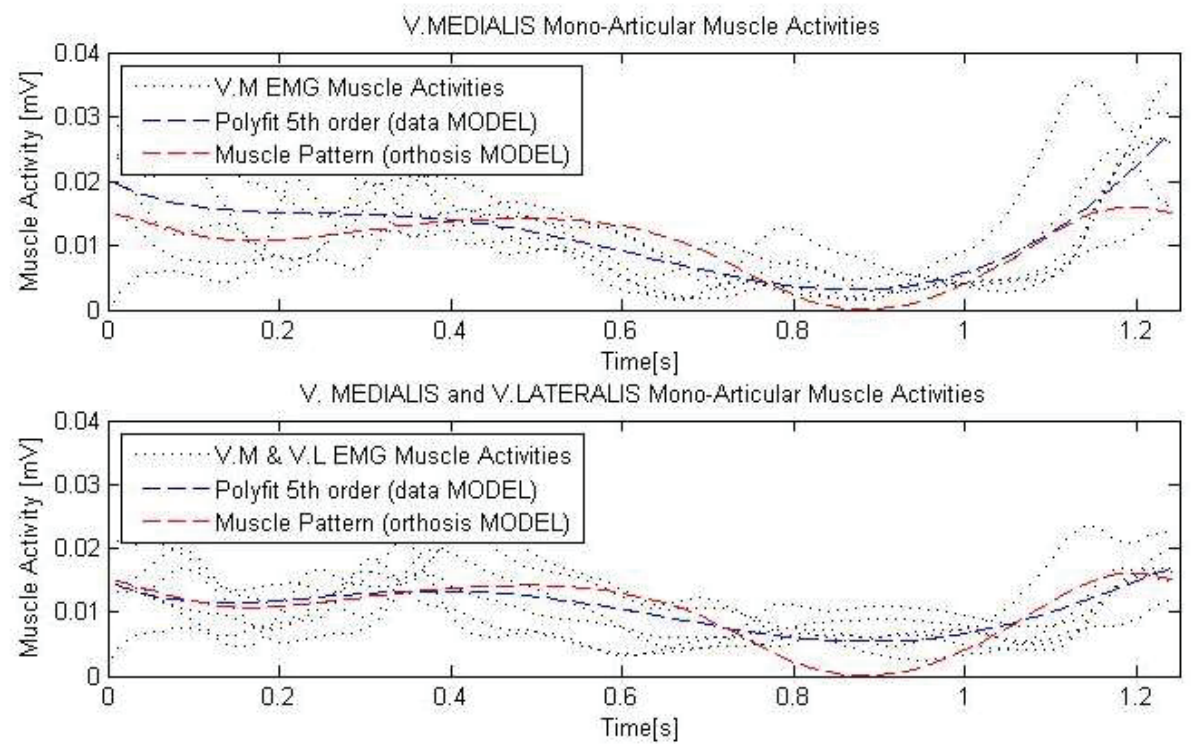

Fig. 2. Evaluation of the mono-articular muscle's mathematical model with the Vastus Medialis (VM) and Vastus Lateralis (VL) muscle activities. 


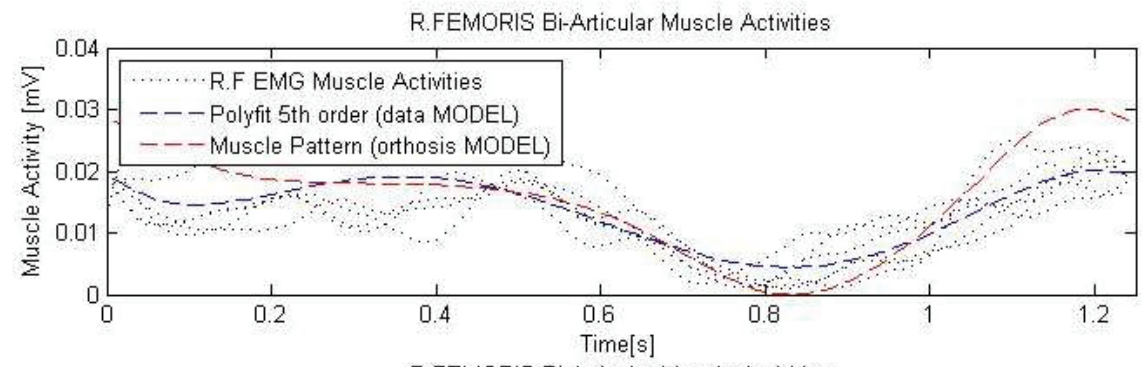

B.FEMORIS Bi-Articular Muscle Activities

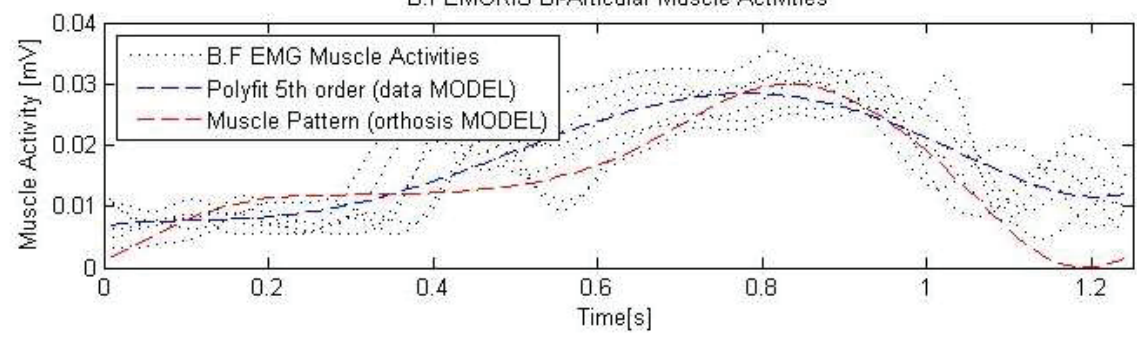

Fig. 3. Evaluation of the bi-articular muscle's mathematical model with the Rectus Femoris (RF) and Bicep Femoris (BF) muscle activities.

The correlation tests between the sEMG muscle activities for VM, VL, RF, and BF muscles models (data MODEL) with derivation models (orthosis MODEL) after rendering the existing delay produced high correlation coefficient values. The correlation value of the antagonistic mono-articular muscles varies between $r=0.83$ for the VM muscle activities and $r=0.93$ for the VM-VL muscle activities. On the other hand, the correlation value of the antagonistic bi-articular muscles varies between $r=0.91$ for the RF muscle activities and $r=0.86$ for the BF muscle activities. Therefore, it could be conclude that the proposed derivation models of the antagonistic mono- and bi-articular muscles were suitable and reliable to be implemented into the control scheme and strategy of the pneumatic muscles actuated lower limb orthosis.

\section{Conclusions}

In conclusions, the verification between sEMG muscle activities of VM, VL, RF, and BF muscles with derivation models shows that the antagonistic mono- and bi-articular muscle activities exhibit co-contraction movements in between anterior and posterior muscles, and muscle synergy in between multiple anterior or posterior muscles with respect to specific joints (i.e., hip and knee joints). Therefore, the hypothesis that the antagonistic muscles were activated and contract simultaneously could be implemented. However, there might be appropriate to include some delay as well in-between the activation of the anterior-posterior mono- and bi-articular muscle models. Based on the verification of the sEMG muscle activities data and its correlation values, it could be concluded that the proposed derivation models of the antagonistic mono- and bi-articular muscles were suitable to simulate and controls the pneumatic muscles actuated lower limb orthosis. Finally, it is crucial to obtain a good derivation model for the antagonistic mono- and bi-articular muscle models to successfully simulate and control the pneumatic muscle actuated lower limb orthosis comparable to human musculoskeletal system. In the future, an actual model of the pneumatic muscle actuated lower limb orthosis would be implemented. 


\section{Acknowledgements}

This research study was supported by Potential Academic Staff (PAS) research grants Q.J130000.2724.02K16, Universiti Teknologi Malaysia (UTM).

\section{References}

1. M.A.M. Dzahir, S.I. Yamamoto, Recent trends in lower-limb robotic rehabilitation orthosis: control scheme and strategy for pneumatic muscle actuated gait trainers, Robotics, 3(2), 120-148 (2014)

2. B.G. Nascimento, C.B. Vimieiro, D.A. Nagem, M. Pinotti, Hip orthosis powered by pneumatic artificial muscle: Voluntary activation in absence of myoelectrical signal, Artif. Organs, 32, 317-322 (2008)

3. C.B.S. Vimieiro, B.G. Nascimento, D.A.P Nagem, M. Pinotti, Development of a hip orthosis using pneumatic artificial muscles, In Proceeding of TMSi, (2005) July18-1; São Paulo, Spain

4. K. Bharadwaj, T.G. Sugar, Kinematics of a robotic gait trainer for stroke rehabilitation, In Proceedings of the IEEE International Conference on Robotics and Automation, (2006) May 15-19; Orlando, FL, USA

5. D.P. Ferris, K.E. Gordon, G.S. Sawicki, A. Peethambaran, An improved powered ankle-foot orthosis using proportional myoelectric control, Gait Posture 23, 425-428 (2006)

6. D.P. Ferris, J.M. Czerniecki, B. Hannaford, An ankle-foot orthosis powered by artificial pneumatic muscles, J. Appl. Biomechanics, 21, 189-197 (2005)

7. K.E. Gordon, G.S. Sawicki, D.P. Fessis, Mechanical performance of artificial pneumatic muscles to power an ankle-foot orthosis, J. Biomech., 39, 1832-1841 (2006)

8. N. Costa, M. Bezdicek, M. Brown, J.O. Gray, D.G. Caldwell, Joint motion control of a powered lower limb orthosis for rehabilitation, Int. J. Autom. Computation, 3, 271-281 (2006)

9. T. Miyoshi, K. Hiramatsu, S.I. Yamamoto, K. Nakazawa, M. Akai, Robotic gait trainer in water: Development of an underwater gait-training orthosis, Disabil. Rehabilitation, 30, 81-87 (2008)

10. P. Malcom, P. Fiers, V. Segers, I. Caekenberghe, M. Lenoir, D. Clercq, Experimental study on the role of the ankle push off in the walk-to-run transition by means of a powered ankle-foot-exoskeleton, Gait Posture, 30, 322-327 (2009)

11. P. Malcom, V. Segers, I. Caekenberghe, D. Clercq, Experimental study of the influence of the $\mathrm{m}$. tibialis anterior on the walk-to-run transition by means of a powered anklefoot-exoskeleton, Gait Posture, 29, 6-10 (2009)

12. S. Galle, P. Malcom, W. Derave, D. Clercq, Adaptation to walking with an exoskeleton that assists ankle extension, Gait Posture, 38, 495-499 (2013)

13. P. Malcom, W. Derave, S. Galle, D. Clercq, A simple exoskeleton that assist plantarflexion can reduce the metabolic cost of human walking, PLoS One, 8, 0056137 (2013)

14. G.S. Sawicki, D.P. Fessis, A pneumatically powered knee-ankle-foot orthosis (KAFO) with myoelectric activation and inhibition, J. Neuro-Eng. Rehabil., 6, 23:1-23:16 (2009)

15. T.T. Deaconescu, A.I. Deaconescu, Pneumatic muscle actuated equipment for continuous passive motion, IAENG Trans. Eng. Technol., doi:10.1063/1.3256258 (2009) 
16. B. Pieter, V.D. Michael, V.H. Ronald, V. Bram, L. Dirk, Design and control of lower limb exoskeleton for robot-assisted gait training, Applied Bionics and Biomechanics, 6(2), 229-243 (2009)

17. B. Pieter, V.D. Michael, V.H. Ronald, V. Bram, L. Dirk, Pleated pneumatic artificial muscle based actuator system as a torque source for compliant lower limb exoskeletons, IEEE/ASME Transactions on Mechatronics, 19(3), 1046-1056 (2014)

18. T.J. Yeh, M.J. Wu, T.J. Lu, F.K. Wu, C.R. Huang, Control of McKibben pneumatic muscles for a power-assist, lower-limb orthosis, Mechatronics, 20, 686-697 (2010)

19. J. Carberry, G. Hinchly, J. Buckerfield, E. Taylor, T. Burton, S. Madgwick, R. Vaidyanathan, Parametric design of an active ankle foot orthosis with passive compliance, In Proceedings of the Computer-Based Medical System (CBMS), (2011) June 27-30; Bristol, UK

20. Y. Park, B. Chen, D. Young, L. Stirling, R. Wood, E. Goldfield, R. Nagpal, Bioinspired Active Soft Orthotic Device for Ankle Foot Pathologies, In Proceedings of the International Conference on Robots and Systems (IROS), (2011) September 25-30; San Francisco, CA, USA

21. Y. Park, B. Chen, C. Majidi, R. Wood, R. Nagpal, E. Goldfield, Active Modular Elastomer Sleeve for Soft Wearable Assistance Robots, In Proceedings of the IEEE International Conference on Robots and Systems (IROS), (2012) October 7-12; Vilamoura, Portugal

22. C.M. Teng, Z.Y. Wong, W.Y. The, Y.Z. Chong, Design and development of inexpensive pneumatically-powered assisted knee-ankle-foot orthosis for gait rehabilitation-preliminary finding, In Proceedings of the International Conference on Biomedical Engineering (ICoBE), (2012) February 27-28; Penang, Malaysia

23. T. Kawamura, K. Takanaka, Development of an orthosis for walking assistance using pneumatic artificial muscle-a quantitative assessment of the effect of assistance, In Proceedings of the International Conference on Rehabilitation Robotics, (2013) June 24-26; Seattle, WA, USA

24. S. Hussain, S.Q. Xie, P.K. Jamwal, Adaptive impedance control of a robotic orthosis for gait rehabilitation, IEEE Trans. Cybern., 43, 1025-1034 (2013)

25. S. Hussain, S.Q. Xie, P.K. Jamwal, Robust nonlinear control of an intrinsically compliant robotic gait training orthosis, IEEE Trans. Syst. Man Cybern.: System, 43, 655-665 (2013)

26. K.D. William, Task-based methods for evaluating electrically stimulated antagonist muscle controllers, IEEE Trans. on Biomedical Engineering, 36 (1989)

27. M. Samer, F. Philippe, G. David, P. Philippe, E.M. Hassan, Towards a co-contraction muscle control strategy for paraplegics. IEEE Conf. on Decision and Control. (2005)

28. H. Stewart, F. Norm, B. Michael, Muscle co-contraction modulates damping and joint stability in a three-link biomechanical limb, Frontiers in Neurorobotics, 5 (2012)

29. M.A.M. Dzahir, S.I. Yamamoto, Design and Evaluation of the AIRGAIT Exoskeleton: Leg Orthosis Control for Assistive Gait Rehabilitation, Journal of Robotics, vol. 2013, Article ID 535106, 20 pages, doi:10.1155/2013/535106 (2013)

30. P. Flavio, M.A.M. Dzahir, S.I. Yamamoto, Computed-torque method for the control of a 2 DOF orthosis actuated through pneumatic artificial muscles: a specific case for the rehabilitation of the lower limb, Medical Physics, arXiv: 1404.6968 [physics.med-ph] (2014) 